\title{
Who needs mirrors?
}

\author{
Over 40 years ago, a device known as a mirrorless optical parametric oscillator was proposed. \\ Only now has it been demonstrated in the lab. Nature Photonics talked to Carlota Canalias and \\ Valdas Pasiskevicius of KTH, The Royal Institute of Technology in Sweden, to find out how.
}

In your latest work, you built the first mirrorless optical parametric oscillator, or MOPO. What exactly is it?

An optical parametric oscillator (or OPO) is a nonlinear device that oscillates at optical frequencies. Essentially, it converts incoming laser light (a pump wave) into two output waves of lower frequencies (the signal and idler). In conventional OPOs the pump and output waves travel in the same direction, but in our case the signal and idler photons actually travel in opposite directions, one with the pump, the other against the pump, and there is distributed feedback between the two waves. This counterpropagating type of configuration does not need a cavity mirror to establish the oscillations, hence the term 'mirrorless'.

What is the benefit of not having a mirror? Being mirrorless brings a number of advantages. First, there is no need for any surface coatings or alignment, and this makes the device very simple and reliable. Second, the counterpropagating nature of the photon interaction can offer a whole class of useful sources of light.

As with any interaction, energy and momentum must be conserved. In a parametric oscillation, where the signal and idler travel in opposite directions, there is a large momentum mismatch, which has to be compensated for by the nonlinear medium. This imposes strong constraints on the momenta of the generated photons, which translates into greater control over the spectrum of the emitted light, for example the width or spatial coherence of the output beams.

\section{How did you create your MOPO?}

The key to our device lies in engineering the nonlinear medium in just the right way. To compensate for the very large momentum mismatch between signal and idler, you need an extraordinarily large material birefringence (or anisotropy), something that most naturally occurring nonlinear materials cannot offer. We therefore use a ferroelectric nonlinear material and an approach known as quasi phase-matching, which involves periodically changing the

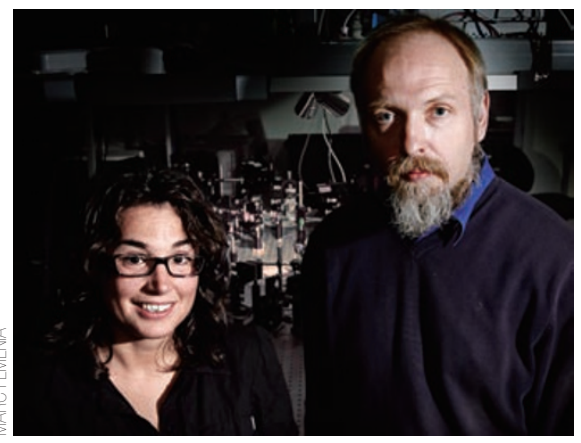

very precise tuning of the output wavelength through temperature control.

The MOPO was proposed theoretically over 40 years ago. Why has it taken so long to be realized?

People have been trying to build MOPOs for many years. Although domain engineering sounds simple, in practice it's not. You have to be able to carefully control all of the domain positions and their aspect ratios, and to do this with domains that are $400 \mathrm{~nm}$ wide, over a device length of several

In the world of MOPOs. Carlota Canalias (left) and Valdas Pasiskevicius (right).

medium's nonlinear optical properties in order to conserve the total momentum in the interaction.

Our material is a rather exotic ferroelectric nonlinear crystal known as KTP (with chemical composition $\mathrm{KTiOPO}_{4}$ ). Using photolithographical patterning and an external electric field, we periodically reverse the ferroelectric domains every $400 \mathrm{~nm}$, a technique known as periodic poling.

\section{What can the device do?}

Our device has special properties that can't be found in normal co-propagating OPOs that use mirrors. With a conventional OPO, the output usually gives a large spectral width and poor spatial coherence of the signal and idler waves. But our MOPO produces a very narrow idler wave (in the mid-infrared) with a bandwidth of about $3 \mathrm{GHz}$, two orders of magnitude narrower than that of the pump, and with a pulse length twice as long. This source can be used for spectroscopy straight away, without any additional narrowing of the output.

Another unique feature of the MOPO is that it imprints the spectral shape of the pump wave onto the signal wave, thereby acting as an excellent frequency converter or controllable wavelength shifter. It is also much more stable to temperature changes than ordinary OPOs, so it can be used for millimetres, is a huge challenge. It requires extensive knowledge of fundamental materials properties and ferroelectricdomain dynamics.

Many efforts have focused on the more popular, cheaper ferroelectric crystal lithium niobate. However, its domains grow much more isotropically than in KTP, making it harder to periodically invert them on a submicrometre scale. If the nonlinear crystal isn't engineered correctly, the oscillation threshold can be too high for parametric oscillation to begin. Not only that, but you have to ensure that the medium has a large nonlinearity, a high damage threshold, and small scattering losses. At KTH, we have been working with KTP for some years and have acquired the experience needed to tune its domains in a very precise way. But it has taken time.

\section{Where next?}

In the future we want to reduce the period of our nanostructured KTP even further. Frequency converters made from ferroelectric or semiconductor materials could lead to new optical devices, such as tunable MOPOs, alloptical switches or spatial soliton generators. Alternatively, the MOPO could be used for generating entangled counterpropagating photon pairs for quantum optics, or as a very tiny broadband parametric amplifier of ultrashort pulses for use in attosecond pulse generation. The possibilities are tantalizing.

Interview by Amber Jenkins.

Canalias and Pasiskevicius have an article on their MOPO on p459 of this issue. 